\title{
Hypoxia-activated prodrugs forge ahead in cancer
}

In February, Merck KGaA delivered a new vote of confidence for hypoxia-inducible prodrugs. On the heels of phase 2 trial success in pancreatic cancer, the Darmstadt, Germany-based pharma signed a deal with Threshold Pharmaceuticals of S. San Francisco, California, worth $\$ 25$ million upfront, a total of $\$ 525$ million in milestones and double-digit royalties to jointly develop and commercialize $\mathrm{TH}-302$, a 2-nitroimidazoletriggered bromo analog of the approved DNA-alkylating agent ifosfamide. Pancreatic cancers have a particularly high rate of hypoxia and $>90 \%$ of tumors bear hypoxic regions. "My guess is that served as the basis for Threshold's deal with Merck," says John Gutheil, CEO of ProActa, a La Jolla, California-based biotech that is currently pursuing another prodrug PR-104, a phosphate precursor of the dinitrobenzamide mustard for treating acute leukemia.

Threshold's phase 2 results, released in March at the American Association for Cancer Research in Chicago, signal a revival of sorts for the prodrug paradigm. In a 214-patient randomized, controlled trial called TC-CR-404, TH-302 in combination with Gemzar (gemcitabine) showed an advantage in median progression-free survival of 5.6 months compared with 3.6 months in patients on Gemzar alone. The response rate was also higher on the prodrug combination: $22 \%$ compared with $12 \%$ on Gemzar alone.

The same month, Threshold also received an orphan drug designation for $\mathrm{TH}-302$ from both European Union (EU; Brussels) and US regulators for soft-tissue sarcoma. Currently in a pivotal phase 3 trial with the Sarcoma Alliance

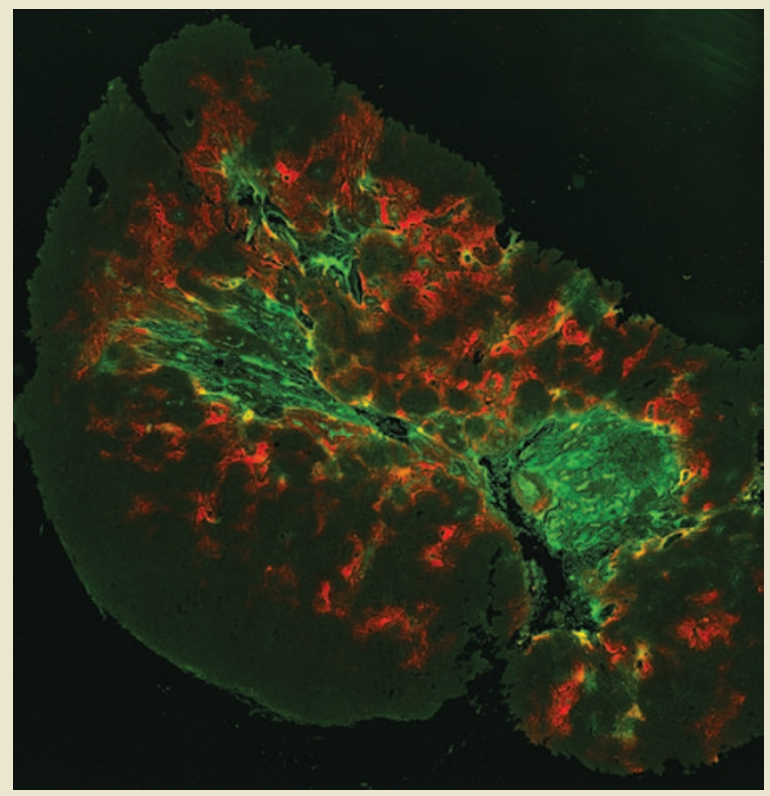

Tumour hypoxia in a human epidermoid cancer xenograft. Hypoxic regions are green and hypoxia probes are red. The central green region is necrotic tissue. Image courtesy of Dr. Maria Abbattista and Dr. Adam Patterson (Translational Therapeutics Team, Auckland Cancer Society Research Centre, University of Auckland, New Zealand). for Research through Collaboration, Threshold retains responsibility to develop $\mathrm{TH}-302$ in this indication.

Over the past 50 years, it has become widely accepted that solid tumors are often hypoxic or oxygen deprived. Tumor hypoxia also causes resistance to chemotherapy and radiotherapy and makes cancers more aggressive and likely to spread. But progress in developing hypoxia-activated drugs has, so far, been stymied by a string of high-profile failures. Of companies developing hypoxia-activated drugs, two have dropped their candidates, including Paris-based Sanofi, which abandoned the drug tirapazamine in 2007 due to poor efficacy in (among others) head and neck cancer, and Novacea, whose small-molecule prodrug, banoxantrone, foundered in 2008 when the S. San Francisco biotech struggled under the weight of other clinical failures in glioblastoma multiforme and B-cell neoplasms.

The concept behind hypoxia-activated prodrugs is that they undergo uncloaking at low oxygen pressure. Prodrugs are inactive when administered and metabolized in vivo. Conversion takes place in two steps. First, a one-electron reductase enzyme creates an oxygen-sensitive intermediate. Second, if the intermediate is in a normal tissue, where oxygen pressure varies between $20 \mathrm{~mm} \mathrm{Hg}$ and $80 \mathrm{~mm} \mathrm{Hg}$, it reoxidizes to the inert prodrug. In the absence of oxygen, the intermediate spontaneously converts to the active drug. Within some tumors, oxygen can plummet to $1 \mathrm{~mm} \mathrm{Hg}$ or below in areas where normal blood vessel architecture is disrupted by the tumor's growth.

But even normal tissue can be hypoxic under certain conditions. In the case of Sanofi's shelved tirapazamine, for instance, the prodrug was converted to the active form at oxygen levels only slightly below those found in normal tissue. This high activation threshold, says ProActa's Gutheil, may have prompted tirapazamine to release the active drug in some normal tissues with low-oxygen regions, giving rise to side effects.

"Getting that right has been one of the challenges," says William Wilson, a professor at the University of Auckland in New Zealand, who developed ProActa's lead compound PR104 , currently in phase $1 / 2$ clinical trials for acute leukemia. PR-104 and TH-302 have more stringent hypoxia thresholds in the range of $1-5 \mathrm{~mm} \mathrm{Hg}$ oxygen, which Gutheil believes will reduce or eliminate off-target activation. Another advantage of recently developed prodrugs is a longer half-life. As hypoxia within tumors fluctuates, discrete regions may become hypoxic at different times. A short-acting drug might hit one hypoxic region but miss another that appears hours later.

Hypoxia within tumors could explain the problems that have plagued angiogenesis inhibitors like Avastin (bevacizumab), says Charles Hart, Threshold's vice president of biology. It has been speculated that hypoxic environments promoted by angiogenesis inhibitors in turn breed more malignant tumor phenotypes. "There's a rational reason why our drug might work well in combination with anti-angiogenic drugs," says Hart. The company is following closely some pilot studies looking at such combinations.

Jim Kling Bellingham, Washington 